\title{
26 Research Square \\ The clinical traits of acute pancreatitis in aging patients: a retrospective analysis of 473 cases
}

Nonghua Lu ( $\square$ lunonghua@ncu.edu.cn )

First Affiliated Hospital of Nanchang University https://orcid.org/0000-0003-4373-551X

Bingjun Yu

first affiliated hospital of nanchang university

Fengwen Xie

first affiliated hospital of nanchang university

Research article

Keywords: Acute pancreatitis, aging patients, clinical traits, death rate, ICU admission rate

Posted Date: February 19th, 2020

DOI: https://doi.org/10.21203/rs.2.24010/v1

License: (1) This work is licensed under a Creative Commons Attribution 4.0 International License.

Read Full License 


\section{Abstract}

\section{Background}

The incidence of acute pancreatitis in aging patients has increasing in recent years. Controversial results about clinical outcomes of acute pancreatitis in aging patients were reported in different literature. The aim of our study was to compare the clinical outcomes of AP in aging patients between 60-79 years old and over 80 years old.

\section{Methods}

80 patients aged $\geq 80$ years old(oldest group) were compared to 393 patients aged 60 to 79 years old(older group). The clinical course, biochemical, radiological data were enrolled. The primary endpoint was to compare the death rate, intensive care unit admission rate and in-hospital length of stay(LOS). The secondary endpoint was operative treatment and the complications of AP.

Results

Abdominal symptom of abdominal pain $(61.3 \%$ vs $46.3 \%, \mathrm{P}=0.013)$ was less in oldest group, while diarrhea(18.3\% vs $30.0 \%, P=0.018$ ), jaundice( $8.9 \%$ vs $17.5 \%, P=0.021)$, dyspnea( $11.5 \%$ vs $26.3 \%$, $\mathrm{P}=0.001)$ were more obvious in older group than oldest group. A higher death rate $(8.9 \%$ vs $16.3 \%, \mathrm{P}=$ $0.003)$ and longer hospital length of stay $(11.51 \pm 10.19$ vs $15.26 \pm 11.04, P=0.001)$ were found in aging patients aged $\geq 80$ years old. Mean BMI was lower in oldest group compared to older group(22.36 \pm 2.89 vs $21.07 \pm 3.18, P=0.001)$. Multivariate analysis identified aged over 80 years(OR $3.299,95 \% \mathrm{Cl} 1.316$ 8.269, $P=0.011)$ and organ failure $(P<0.05)$ as independent risk factors of mortality. More severe of $A P(O R$ $11.722,95 \% \mathrm{Cl} 4.780-28.764, \mathrm{P}=0.001$ ), abdominal pain(OR 1.906, 95\% Cl 1.052-3.453, $\mathrm{P}=0.033$ ) and organ failure $(P<0.05)$ were recognized as influencing intensive care unit rate. Aging patients aged over 80 years old(OR $0.149,95 \% \mathrm{Cl} 2.027-6.268, \mathrm{P}=0.001)$, more severe of $\mathrm{AP}(\mathrm{OR} 0.218,95 \% \mathrm{Cl} 1.567-4.322$, $\mathrm{P}=0.001)$, female(OR 0.093, 95\% Cl 0.336-3.542, $\mathrm{P}=0.018$ ), Jaundice(OR 0.080, 95\% Cl 0.146-5.324, $P=0.038)$, operative treatment $(P<0.05)$ and organ failure $(P<0.05)$ were the risk factors for LOS.

\section{Background}

In Europe, the improvement of medical and health environment resulted an increase of $2.4 \%$ aging people over 65 years in the past decade. More importantly, they predicted that aging people aged over 80 years old is expected to reach the $12.7 \%$ of all people in 2080 as compared to the $5.5 \%$ in $2007[1]$. With the aging of world population trends in the intensification of the aging phenomenon has become a major strategic issue and an increasing number of aging patients suffered to acute pancreatitis(AP)[2-4].

AP is a potentially fatal clinical disease which is characterized by edema, hemorrhage and necrosis[5-7]. The clinical process of AP varies from a self-limiting acute inflammation to a clinically severe condition, potentially leading to life-threatening disease for the risk of multi-organ failure [8]. The death rate of AP is 
extremely different varying from $5 \%$ of mild acute pancreatitis(MAP) $[9,10]$ up to $30 \%$ or more of severe acute pancreatitis(SAP)[11, 12].

AP is a life-threating disease that is certificated by large numbers of researches. The frail population of aging people easily developed to severe condition as compared to younger patients after AP onset. Hence, it is necessary to figure out the outcome and the clinical traits of AP in the increasing part of aging population and recognized a useful medical and interventional treatment in this high risk category is crucial .

The diagnosis of AP in aging patients is more difficult for physician compared to the younger AP patients. The symptoms of aging patients were presented later and have non-specific presentation in the development of the disease[13]. Furthermore, their great number of comorbidities rate may potentially result to severe consequences in such an acute disease[14-17].

However, although age is an element influencing the procession of AP[18-21], its influence on the clinical traits in AP remained controversial. The reason probably due to the presence of only little studies surveyed the clinical traits of AP in aging population. A research reported that aging patients have a more severe course in elderly patients but the mortality rate is not increased[14]. Conversely, other studies proposed that age itself is an independent risk factor for intensive care unit (ICU) admission and mortality $[19,22]$. Actually, age is an vital component utilized in stratified the severity of the disease [8].

These inconsistent results of those research are probably due to the different age partitions to define aging patients by different countries and authors, thus making difficult to compare the clinical outcomes of AP aging patients. Hence, it is necessary to divided the aging patients to a heterogeneous cohort and then analyze the clinical outcomes of AP patients according to the different age subsets.

In our research, we decided to focus our attention on the subgroup of oldest patients group ( $\geq 80$ years) and the older patients group (60-79 years) according to Chinese age standardization of rates. The aim of our study was to investigate the clinical traits of AP in oldest patients group and older patients group like incidence, clinical course, and mortality rate.

\section{Methods}

\section{Patient data}

The retrospective study enrolled all patients with AP from the AP database of the First Affiliated Hospital of Nanchang University, which was approved by the ethics committee (database approval number: 2011001). The study comprised 2148 patients recruited from the AP database from January 1, 2018 to December 31, 2019. Younger than 60 years of AP patients were excluded from the study, as were those more than 7d after AP onset and so on. We eventually enrolled 473 aging patients of AP in our study. The exclusion criterias were as listed in Fig. 1. Clinical data such as age, sex, history of smoking and drinking, Diabetes, hypertension, history of renal diseases, complications, the severity and etiology of AP during 
hospitalization, death, biomarkers of clinical tests, Length of stay in hospital after AP onset, intensive care unit(ICU)admission and body mass index (BMI) on admission and so on were recorded by us. $\mathrm{BMI}(\mathrm{kg} / \mathrm{m} 2)$ was defined as weight in kilograms divided by height in square metres. Acute physiology and chronic health evaluation II (APACHE II) score, Bedside index for severity in acute pancreatitis score, Marshall and Glasgow score were calculated based on patient data.

\section{Diagnosis and definition}

The total population was divided into two groups called the older group and the oldest group. The older group was patients aged from 60 to 80 years old. The oldest group was AP patients aged over 80 years. The diagnosis of AP was based on the 2012 revised Atlanta classification criteria[23]. The 2012 revision of the Atlanta Acute Pancreatitis Diagnostic and Classification Criteria was used to classify the AP severity and etiology. Organ failure was according to the modified Marshall Scoring System[23]. Pancreatic complications was diagnosed by Contrast-enhanced computed tomography (CECT) which indicated by diffuse enlargement of the pancreatic or peripancreatic volume, blurred edges, mild enhancement of the edema area after enhanced scanning, and no enhancement of necrotic areas[10, 24]. The death regarded as death during hospitalization. The definition is shown in Supplementary Table 1. Physical examination was aimed at assessing the patients' general health condition. The actual clinical course, results of radiological (CT or CECT) and laboratory tests (white blood cells(WBC), Lactic dehydrogenase(LDH), Alanine Aminotransferase (ALT), Aspartate transaminase(AST), total bilirubin (TBIL), Direct bilirubin (DBIL), C-reactive protein(CRP), Urea nitrogen(BUN), Glucose(GLU), Total cholesterol(TC), Triglyceride(TG), Albumin(ALB), Amylase(AMY), Creatinine(Cr)) were assessed in due consideration of the respective older group (60-79 years)and oldest group $(\geq 80$ years). Chronic pancreatitis or pancreatic cancer in our study were excluded from further assessment.

\section{Statistical analysis}

Categorical variables were statistically compared by the chi-square test at the univariate analysis. MannWhitney $U$ test was used to continuous variables.

Categorical variables are presented as numbers and percentages, and continuous variables are presented as median and mean difference. 0.05 was regarded as the significance level. Variable significant at univariate and multivariate analysis of logistic regression model were used to distinguish independent factor of mortality, in-hospital length of stay(LOS) and ICU admission rate. Results were expressed as odds ratio (OR) with $95 \%$ confidence interval $(\mathrm{Cl})$. All data were analyzed by SPSS $24 \mathrm{R}$.

\section{Results}

From January 2018 to December 2019, a total of 2148 patients were admitted in the tertiary hospital, the first affiliated hospital of Nanchang university. Four hundred seventy-three aging AP patients were finally included after excluding unqualified patients of AP. The flow chart was listed in Fig. 1. In this cohort, all aging AP patients were divided into two groups, 393 patients in older group aged from 60 to 80 years old, 
80 patients in oldest group aged $\geq 80$ years old. There were 217 males $(45.9 \%)$ and 256 females $(54.1 \%)$ in the enrolled aging AP patients. The mean age was $70.46 \pm 7.67$ years of all the AP patients, $67.82 \pm$ 5.26 in older group and $83.46 \pm 2.81$ in oldest group. Length of stay in hospital in oldest group(15.26 \pm $11.04)$ are longer than older group $(11.51 \pm 10.19)$. There were $129(32.8 \%)$ patients in older group and $30(37.5 \%)$ in oldest group of intensive care unit(ICU)admission. The mean BMI in older group $(21.07 \pm$ 3.18) were less than oldest group $(22.36 \pm 2.89)(P=0.001)$. The detailed data are listed in Table 1 . The APAPCHEll score in oldest group $(13.08 \pm 3.50)$ is higher than older group $(11.91 \pm 3.74)$. There was significant different in the two groups $(p=0.01)$. Other scores were also listed in Table 2 . History of smoking and drinking, Chronic renal failure, hypertension, diabetes, severity of AP and incidence of complication were presented in Table 1 as baseline characteristics. According to the biochemical values, there were no differences in the older group and oldest group including white blood cell (WBC), serum amylase, C-reactive protein(CRP) and so on as listed in Table 2. 
Table 1

Clinical characteristics of AP patients in the older group and oldest group.

\begin{tabular}{|c|c|c|c|c|}
\hline & $\begin{array}{l}\text { All patients(n } \\
=473)\end{array}$ & $\begin{array}{l}\text { Older group }(60-80 \text { years } \\
)(n=393)\end{array}$ & $\begin{array}{l}\text { Oldest group }(\geq \\
80 \text { years })(n=80)\end{array}$ & $\begin{array}{l}\mathrm{P} \\
\text { value }\end{array}$ \\
\hline Age(years) & $70.46 \pm 7.67$ & $67.82 \pm 5.26$ & $83.46 \pm 2.81$ & 0.001 \\
\hline \multicolumn{5}{|l|}{ Sex } \\
\hline Male & $217(45.9 \%)$ & $180(45.8 \%)$ & $37(46.3 \%)$ & 0.942 \\
\hline Female & $256(54.1 \%)$ & $213(54.2 \%)$ & $43(53.8 \%)$ & \\
\hline BMI & $22.14 \pm 2.98$ & $22.36 \pm 2.89$ & $21.07 \pm 3.18$ & 0.001 \\
\hline \multicolumn{5}{|c|}{ Severity of AP } \\
\hline MAP & $150(31.7 \%)$ & $119(30.3 \%)$ & $31(38.8 \%)$ & 0.312 \\
\hline MSAP & $190(40.2 \%)$ & $160(40.7 \%)$ & $30(37.5 \%)$ & \\
\hline SAP & $133(28.1 \%)$ & $114(29.0 \%)$ & 19(23.8\%) & \\
\hline \multicolumn{5}{|c|}{ Etiology of AP } \\
\hline Biliary & $384(81.2 \%)$ & $313(79.6 \%)$ & $71(88.6 \%)$ & 0.203 \\
\hline HTG & $17(3.6 \%)$ & $16(4.1 \%)$ & $1(1.3 \%)$ & \\
\hline Alcohol & $20(4.2 \%)$ & $19(4.8 \%)$ & $1(1.3 \%)$ & \\
\hline Others & $52(11.0 \%)$ & $45(11.5 \%)$ & $7(8.8 \%)$ & \\
\hline \multicolumn{5}{|c|}{ Hypertension } \\
\hline Yes & $169(35.7 \%)$ & 134(34.1\%) & $35(43.8 \%)$ & 0.101 \\
\hline No & $304(64.3 \%)$ & $259(65.9 \%)$ & $45(56.3 \%)$ & \\
\hline \multicolumn{5}{|l|}{ Diabetes } \\
\hline Yes & $37(7.8 \%)$ & $31(92.1 \%)$ & $6(7.5 \%)$ & 0.906 \\
\hline No & $436(92.2 \%)$ & $362(92.1 \%)$ & $74(92.5 \%)$ & \\
\hline
\end{tabular}

For continuous variables are presented as mean (standard deviation), categorical variables are presented as number (percentage).

Abbreviation: Acute pancreatitis $=$ AP. Acute respiratory failure $=$ ARF. Length of stay $=$ LOS. Endoscopic retrograde cholangiopancreatography $=$ ERCP. Intensive care Unit $=$ ICU. Body mass index $=$ BMI. Acute kidney injury = AKI. Mild acute pancreatitis = MAP. Moderately severe acute pancreatitis = MSAP. Severe acute pancreatitis $=$ SAP. Hypertriglyceridemia $=$ HTG. Endoscopic retrograde cholangiopancreatography $=$ ERCP. Pancreatic pseudocyst $=$ PPC. Walled-off necrosis $=$ WON. Acute peripancreatic fluid collection $=$ APFC. Acute necrosis collection $=$ ANC. Percutaneous drainage $=$ PCD . Hypertriglyceridemia $=$ HTG 


\section{All patients(n Older group(60-80 years Oldest group $(\geq$ \\ = 473) $\quad(n=393)$ \\ 80 years $)(n=80)$ \\ P \\ value}

Chronic renal

failure

$\begin{array}{lllll}\text { Yes } & 11(2.3 \%) & 10(2.5 \%) & 79(98.8 \%) & 0.484 \\ \text { No } & 462(97.7 \%) & 383(97.5 \%) & 1(1.3 \%) & \end{array}$

History of

smoking

$\begin{array}{lllll}\text { Yes } & 101(21.4 \%) & 90(22.9 \%) & 11(13.8 \%) & 0.069 \\ \text { No } & 372(78.6 \%) & 303(77.1 \%) & 69(86.3 \%) & \end{array}$

History of

drinking

$\begin{array}{lllll}\text { Yes } & 84(17.8 \%) & 73(18.6 \%) & 11(13.8 \%) & 0.303 \\ \text { No } & 389(82.2 \%) & 320(81.4 \%) & 69(86.3 \%) & \end{array}$

Complication

Organ failure

\begin{tabular}{lllll}
$\begin{array}{l}\text { Acute respiratory } \\
\text { failure }\end{array}$ & $120(25.4 \%)$ & $102(26.0 \%)$ & $18(22.5 \%)$ & 0.517 \\
\hline Acute renal failure & $57(12.1 \%)$ & $44(11.2 \%)$ & $13(16.3 \%)$ & 0.206 \\
\hline Shock & $23(4.9 \%)$ & $22(5.6 \%)$ & $1(1.3 \%)$ & 0.099 \\
$\begin{array}{l}\text { Multi-organ } \\
\text { failure }\end{array}$ & $45(9.5 \%)$ & $35(8.9 \%)$ & $10(12.5 \%)$ & 0.318 \\
\hline
\end{tabular}

Pancreatic

complications

$\begin{array}{lllll}\text { ANC } & 31(6.6 \%) & 26(6.6 \%) & 5(6.3 \%) & 0.073 \\ \text { ANC infection } & 28(5.9 \%) & 26(6.6 \%) & 2(2.5 \%) & \\ \text { APFC } & 123(26.0 \%) & 108(27.5 \%) & 15(18.8 \%)\end{array}$

For continuous variables are presented as mean (standard deviation), categorical variables are presented as number (percentage).

Abbreviation: Acute pancreatitis $=$ AP. Acute respiratory failure $=$ ARF. Length of stay $=$ LOS. Endoscopic retrograde cholangiopancreatography $=$ ERCP. Intensive care Unit $=$ ICU. Body mass index $=$ BMI. Acute kidney injury = AKI. Mild acute pancreatitis = MAP. Moderately severe acute pancreatitis = MSAP. Severe acute pancreatitis = SAP. Hypertriglyceridemia = HTG. Endoscopic retrograde cholangiopancreatography $=$ ERCP. Pancreatic pseudocyst $=$ PPC. Walled-off necrosis $=$ WON. Acute peripancreatic fluid collection $=$ APFC. Acute necrosis collection $=$ ANC. Percutaneous drainage $=$ PCD. Hypertriglyceridemia $=$ HTG 


\begin{tabular}{|c|c|c|c|c|}
\hline & $\begin{array}{l}\text { All patients(n } \\
=473)\end{array}$ & $\begin{array}{l}\text { Older group }(60-80 \text { years } \\
)(n=393)\end{array}$ & $\begin{array}{l}\text { Oldest group }(\geq \\
80 \text { years })(n=80)\end{array}$ & $\begin{array}{l}P \\
\text { value }\end{array}$ \\
\hline APFC infection & $14(3.0 \%)$ & $13(3.3 \%)$ & $1(1.3 \%)$ & \\
\hline WON & $6(1.3 \%)$ & $4(1.3 \%)$ & $2(2.5 \%)$ & \\
\hline WON infection & $4(0.8 \%)$ & $2(0.5 \%)$ & $2(2.5 \%)$ & \\
\hline PPC & $2(0.4 \%)$ & $1(0.3 \%)$ & $1(1.3 \%)$ & \\
\hline \multicolumn{5}{|l|}{$\begin{array}{l}\text { Operative } \\
\text { treatment }\end{array}$} \\
\hline ERCP & $103(21.8 \%)$ & $81(20.6 \%)$ & $22(27.5 \%)$ & 0.174 \\
\hline PCD & $117(24.7 \%)$ & $93(23.7 \%)$ & $24(30.0 \%)$ & 0.231 \\
\hline Cholecystectomy & $19(4.0 \%)$ & $19(4.8 \%)$ & $0(0.0 \%)$ & 0.097 \\
\hline Necrosectomy & $32(6.8 \%)$ & $28(7.1 \%)$ & $4(5.0 \%)$ & 0.097 \\
\hline \multicolumn{5}{|l|}{ Symptoms } \\
\hline Abdominal pain & $278(58.8 \%)$ & $241(61.3 \%)$ & $37(46.3 \%)$ & 0.013 \\
\hline Vomiting & $34(7.2 \%)$ & $26(6.6 \%)$ & $8(10 \%)$ & 0.285 \\
\hline Diarrhea & $96(20.3 \%)$ & $72(18.3 \%)$ & $24(30.0 \%)$ & 0.018 \\
\hline Jaundice & $49(10.4 \%)$ & $35(8.9 \%)$ & $14(17.5 \%)$ & 0.021 \\
\hline Dyspnea & $66(14.0 \%)$ & $45(11.5 \%)$ & $21(26.3 \%)$ & 0.001 \\
\hline \multicolumn{5}{|l|}{ ICU admission } \\
\hline Yes & $159(33.6 \%)$ & $129(32.8 \%)$ & $30(37.5 \%)$ & 0.42 \\
\hline No & $314(66.4 \%)$ & $264(67.2 \%)$ & $50(62.5 \%)$ & \\
\hline LOS, days & $12.14 \pm 10.42$ & $11.51 \pm 10.19$ & $15.26 \pm 11.04$ & 0.003 \\
\hline \multicolumn{5}{|l|}{ Death } \\
\hline Yes & $48(10.1 \%)$ & $35(8.9 \%)$ & $13(16.3 \%)$ & 0.047 \\
\hline
\end{tabular}

For continuous variables are presented as mean (standard deviation), categorical variables are presented as number (percentage).

Abbreviation: Acute pancreatitis $=$ AP. Acute respiratory failure $=$ ARF. Length of stay $=$ LOS. Endoscopic retrograde cholangiopancreatography $=$ ERCP. Intensive care Unit $=$ ICU. Body mass index $=$ BMI. Acute kidney injury = AKI. Mild acute pancreatitis = MAP. Moderately severe acute pancreatitis = MSAP. Severe acute pancreatitis $=$ SAP. Hypertriglyceridemia $=$ HTG. Endoscopic retrograde cholangiopancreatography $=$ ERCP. Pancreatic pseudocyst $=$ PPC. Walled-off necrosis $=$ WON. Acute peripancreatic fluid collection $=$ APFC. Acute necrosis collection $=$ ANC. Percutaneous drainage $=$ PCD . Hypertriglyceridemia $=$ HTG 


\begin{tabular}{|lllll|}
\hline $\begin{array}{l}\text { All patients(n } \\
\text { = 473) }\end{array}$ & $\begin{array}{l}\text { Older group }(60-80 \text { years } \\
)(n=393)\end{array}$ & $\begin{array}{l}\text { Oldest group }(\geq \\
\mathbf{8 0} \text { years })(n=80)\end{array}$ & $\begin{array}{l}P \\
\text { value }\end{array}$ \\
\hline No & $425(89.9 \%)$ & $358(91.1 \%)$ & $67(83.8 \%)$ & \\
\hline
\end{tabular}

For continuous variables are presented as mean (standard deviation), categorical variables are presented as number (percentage).

Abbreviation: Acute pancreatitis $=$ AP. Acute respiratory failure $=$ ARF. Length of stay $=$ LOS. Endoscopic retrograde cholangiopancreatography $=$ ERCP. Intensive care Unit $=$ ICU. Body mass index = BMI. Acute kidney injury = AKI. Mild acute pancreatitis = MAP. Moderately severe acute pancreatitis = MSAP. Severe acute pancreatitis $=$ SAP. Hypertriglyceridemia $=$ HTG. Endoscopic retrograde cholangiopancreatography $=$ ERCP. Pancreatic pseudocyst $=$ PPC. . Walled-off necrosis $=$ WON. Acute peripancreatic fluid collection $=$ APFC. Acute necrosis collection $=$ ANC. Percutaneous drainage $=$ PCD. Hypertriglyceridemia $=$ HTG 
Table 2

Comparison of the laboratory tests and score of AP patients in the older group and oldest group.

\begin{tabular}{|c|c|c|c|c|}
\hline & $\begin{array}{l}\text { All AP patients(n } \\
=473)\end{array}$ & $\begin{array}{l}\text { Older group }(60-80 \text { years }) \\
(n=393)\end{array}$ & $\begin{array}{l}\text { Oldest group }(\geq 80 \text { years }) \\
(n=80)\end{array}$ & $\begin{array}{l}P \\
\text { value }\end{array}$ \\
\hline WBC & $11.88 \pm 5.67$ & $11.98 \pm 5.66$ & $11.40 \pm 5.76$ & 0.407 \\
\hline $\mathrm{LDH}$ & $413.13 \pm 307.27$ & $421.20 \pm 325.09$ & $373.48 \pm 194.37$ & 0.206 \\
\hline AST & $90.00 \pm 123.39$ & $90.87 \pm 123.78$ & $85.76 \pm 122.16$ & 0.736 \\
\hline ALT & $97.95 \pm 125.25$ & $102 \pm 132.23$ & $75.93 \pm 89.35$ & 0.085 \\
\hline ALB & $35.30 \pm 4.94$ & $34.92 \pm 4.55$ & $35.37 \pm 5.02$ & 0.459 \\
\hline AMY & $496.39 \pm 593.55$ & $501.04 \pm 599.08$ & $473.52 \pm 568.69$ & 0.706 \\
\hline GLU & $8.35 \pm 4.43$ & $8.32 \pm 4.27$ & $8.48 \pm 5.18$ & 0.771 \\
\hline TG & $1.41 \pm 2.85$ & $1.49 \pm 3.11$ & $1.06 \pm 0.62$ & 0.219 \\
\hline TC & $3.92 \pm 1.59$ & $3.95 \pm 1.68$ & $3.78 \pm 0.99$ & 0.409 \\
\hline TBIL & $34.39 \pm 34.73$ & $34.30 \pm 34.80$ & $34.83 \pm 34.48$ & 0.902 \\
\hline DBIL & $20.35 \pm 30.91$ & $20.23 \pm 31.36$ & $20.92 \pm 28.78$ & 0.857 \\
\hline BUN & $7.21 \pm 5.85$ & $7.21 \pm 6.07$ & $7.18 \pm 4.70$ & 0.958 \\
\hline $\mathrm{Cr}$ & $88.63 \pm 76.66$ & $88.06 \pm 79.63$ & $91.45 \pm 60.33$ & 0.719 \\
\hline CRP & $122.57 \pm 94.60$ & $126.22 \pm 96.52$ & $104.64 \pm 82.79$ & 0.063 \\
\hline APACHEII & $12.10 \pm 3.72$ & $11.91 \pm 3.74$ & $13.08 \pm 3.50$ & 0.01 \\
\hline $\begin{array}{l}\text { APACHEII- } \\
\text { age }\end{array}$ & $7.38 \pm 3.58$ & $7.43 \pm 3.60$ & $7.10 \pm 3.49$ & 0.443 \\
\hline BISAP & $1.93 \pm 1.15$ & $1.90 \pm 1.15$ & $2.05 \pm 1.16$ & 0.317 \\
\hline $\begin{array}{l}\text { BISAP- } \\
\text { age }\end{array}$ & $0.99 \pm 1.10$ & $0.98 \pm 1.09$ & $1.06 \pm 1.15$ & 0.567 \\
\hline Marshall & $1.36 \pm 1.25$ & $1.36 \pm 1.27$ & $1.38 \pm 1.15$ & 0.379 \\
\hline Glasgow & $0.06 \pm 0.30$ & $0.48 \pm 0.28$ & $0.10 \pm 0.37$ & 0.156 \\
\hline \multicolumn{5}{|c|}{ For continuous variables are presented as mean (standard deviation). } \\
\hline \multicolumn{5}{|c|}{$\begin{array}{l}\text { Abbreviation: Acute pancreatitis = AP. White blood cell = WBC. Lactic dehydrogenase }=\text { LDH. Alanine } \\
\text { Aminotransferase }=\text { ALT. Aspartate transaminase }=\text { AST. Total bilirubin }=\text { TBIL. Direct bilirubin }=\text { DBIL. } \\
\text { C-reactive protein = CRP. Urea nitrogen }=\text { BUN. Glucose }=\text { GLU. Total cholesterol }=\text { TC. Triglyceride = TG. } \\
\text { Albumin = ALB. Amylase = AMY. Creatinine }=\text { Cr. Acute Physiology and Chronic Health Evaluation II = } \\
\text { APACHEII. Bedside index for severity in acute pancreatitis = BISAP. Hypertriglyceridemia }=\text { HTG }\end{array}$} \\
\hline
\end{tabular}


Various clinical symptoms were presented in AP patients. In our study, we found that abdominal pain was more frequently occurred in the older group than oldest group (241 patients (61.3\%) vs. 37 patients $(46.3 \%))$. While the symptoms of diarrhea, jaundice, dyspnea were more often happened in oldest AP patients. While symptoms of vomiting emerged an approximately similar incidence in the older and oldest group (26 patients (6.6\%) vs. 8 patients (10.0\%)). Meanwhile, the symptoms of diarrhea, jaundice, dyspnea were always accompanying abdominal pain in aging AP patients. The details were listed in Table 1.

The main etiology of AP among the aging patients was biliary ( $81.2 \%$ in aging patients). The similar incidence of the etiology of gallstones of AP were verified in the oldest group(71 patients (88.6\%)) and older group(313 patients (79.6\%)). Alcohol, hypertriglyceridemia, and other type of AP were also listed in Table 1.

According to the contrast-enhanced computerized tomography (CECT), Acute necrosis collection (ANC) was detected in $26(6.6 \%)$ patients of the older group as compared to $5(6.3 \%)$ patients of the oldest cohort. Acute peripancreatic fluid collection (APFC), a more common pancreatic complication, presented a large proportion of rate in the two aging group (108 and 15 in the older and oldest cohorts, respectively). Walled-off necrosis(WON) was found in $4(1.3 \%)$ and $2(2.5 \%)$ patients of the older group and oldest group, respectively. The infection of ANC, APFC and WON seldomly occurred and there were on significant different in aging patients.

Operative treatment is one of the mean of the AP treatment. Endoscopic retrograde cholangiopancreatography (ERCP) was equally used in the two aging groups (81(20.6\%) older group and 22(27.5\%) oldest patients, $P=0.174)$. Percutaneous drainage $(P C D)$ was performed a litter more in oldest patients compared to older group(93(23.7\%)older patients vs. 24(30.0\%)oldest patients), while there were no different in the two group $(P=0.231)$. At the same time, we found that necrosectomy was operated in $28(7.1 \%)$ AP patients in older group and $4(5.0 \%)$ in oldest group. Interestingly, Cholecystectomy was only performed in older group(19(4.8\%)) as compared to oldest group $0(0.0 \%)$.

Oldest group was longer length of stay of hospitalization than older group(11.51 \pm 10.19 vs $11.51 \pm 10.19$, $P=0.003)$. Conversely, no difference was evidenced between the two group of the admitted to ICU (129(32.8\%) older group vs. 30(37.5\%) oldest group). Death rate was higher in the oldest group $(13(16.3 \%))$ as compared to older group $(35(8.9 \%)$ and there were different in the two group $(P=0.047)$. Multiple organ failure was more frequent in oldest group than the older group (10(12.5\%) vs $35(8.9 \%)$ ). There were $18(22.5 \%)$ in older group and $102(26.0 \%)$ in oldest group of acute respiratory failure. The acute renal failure was $13(16.3 \%)$ in oldest group and $44(11.2 \%)$ in the older group. It is surprised that 22(5.6\%) older patients occurred Shock while 1(1.3\%) patients in older group. All of the organ failure were no different in our study. 


\section{Univariate and multivariate regression analysis of risk factors of clinical outcomes}

Death Rate (The Details Were Listed In Table 3) 
Table 3

Univariate and multivariate analysis of the risk factors on mortality in aging patients of acute pancreatitis

\begin{tabular}{|lllll|}
\hline Variable & Univariate analysis & & Multivariate analysis & \\
\hline Age(years) & OR $(95 \% \mathrm{Cl})$ & P value & OR $(95 \% \mathrm{Cl})$ & P value \\
\hline Sex Male/ Female & $1.058(1.019-1.098)$ & 0.003 & $3.299(1.316-8.269)$ & 0.011 \\
\hline BMI & $1.058(1.019-1.098)$ & 0.546 & & \\
\hline Severity of AP & $1.034(0.936-1.142)$ & 0.512 & & \\
\hline MAP & & & & \\
\hline MSAP & Reference & & Reference & \\
\hline SAP & $2.722(0.736-10.074)$ & 0.134 & $1.578(0.354-7.117)$ & 0.346 \\
\hline Etiology of AP & $17.500(5.237-58.479)$ & 0.001 & $2.372(0.453-12.426)$ & \\
\hline Biliary & & & & \\
\hline HTG & Reference & & & \\
\hline Alcohol & $0.553(0.071-4.283)$ & 0.570 & & \\
\hline Others & $1.561(0.438-5.566)$ & 0.492 & & \\
\hline Hypertension & $1.314(0.705-2.448)$ & 0.389 & & \\
\hline Diabetes & $1.353(0.675-2.713)$ & 0.394 & & \\
\hline Chronic renal failure & $0.883(0.111-7.051)$ & 0.907 & & \\
\hline History of smoking & $1.107(0.543-2.256)$ & 0.780 & & \\
\hline History of drinking & $1.434(0.699-2.941)$ & 0.326 & & \\
\hline Symptoms & $1.353(0.675-2.713)$ & 0.394 & & \\
\hline Abdominal pain & $0.484(0.113-2.081)$ & 0.330 & & \\
\hline Vomiting & & & & \\
\hline Diarrhea & & & & \\
\hline
\end{tabular}

Abbreviation: Acute pancreatitis $=$ AP. Acute respiratory failure $=$ ARF. Length of stay $=$ LOS. Endoscopic retrograde cholangiopancreatography $=$ ERCP. Intensive care Unit $=$ ICU. Body mass index $=$ BMI. Acute kidney injury = AKI. Mild acute pancreatitis = MAP. Moderately severe acute pancreatitis = MSAP. Severe acute pancreatitis $=$ SAP. Odds ratio $=$ OR. Endoscopic retrograde cholangiopancreatography $=$ ERCP. Pancreatic pseudocyst $=$ PPC. Walled-off necrosis $=$ WON. Acute peripancreatic fluid collection $=$ APFC. Acute necrosis collection $=$ ANC. Percutaneous drainage $=$ PCD . Hypertriglyceridemia $=$ HTG 


\begin{tabular}{|c|c|c|c|c|}
\hline Variable & \multicolumn{2}{|l|}{ Univariate analysis } & \multicolumn{2}{|l|}{ Multivariate analysis } \\
\hline Jaundice & $1.269(0.519-3.158)$ & 0.608 & & \\
\hline Dyspnea & $1.266(0.564-2.839)$ & 0.568 & & \\
\hline \multicolumn{5}{|l|}{ Operative treatment } \\
\hline ERCP & $0.217(0.564-2.839)$ & 0.012 & $0.284(0.072-1.121)$ & 0.072 \\
\hline PCD & $1.146(0.584-2.249)$ & 0.691 & & \\
\hline Cholecystectomy & $0.000(0.000-)$ & 0.998 & & \\
\hline Necrosectomy & $1.289(0.432-3.845)$ & 0.649 & & \\
\hline \multicolumn{5}{|l|}{ Organ failure } \\
\hline Acute respiratory failure & $13.860(6.783-28.319)$ & 0.001 & $4.226(1.323-13.500)$ & 0.015 \\
\hline Acute renal failure & $15.021(7.646-29.510)$ & 0.001 & $0.943(0.269-3.305)$ & 0.927 \\
\hline Shock & $23.693(9.363-59.954)$ & 0.001 & $4.952(1.575-15.569)$ & 0.006 \\
\hline Multi-organ failure & $22.011(10.685-45.340)$ & 0.001 & $4.612(1.387-15.336)$ & 0.013 \\
\hline \multicolumn{5}{|l|}{ Local complication } \\
\hline ANC & $5.797(2.223-15.117)$ & 0.001 & $0.870(0.231-3.280)$ & 0.837 \\
\hline ANC infection & $9.259(3.645-23.520)$ & 0.001 & $0.881(0.312-2.492)$ & 0.812 \\
\hline APFC & $1.802(0.817-3.975)$ & 0.145 & $0.457(0.039-5.334)$ & 0.532 \\
\hline APFC infection & $1.282(0.157-10.466)$ & 0.817 & $1.350(0.362-5.038)$ & 0.655 \\
\hline WON & $3.333(0.366-30.366)$ & 0.285 & $1.258(0.089-17.770)$ & 0.865 \\
\hline WON infection & $5.556(0.545-56.666)$ & 0.148 & $6.701(0.500-89.739)$ & 0.151 \\
\hline PPC & $0.000(0.000-)$ & 0.999 & $0.000(0.000-)$ & 0.999 \\
\hline \multicolumn{5}{|c|}{$\begin{array}{l}\text { Abbreviation: Acute pancreatitis = AP. Acute respiratory failure }=\text { ARF. Length of stay }=\text { LOS. } \\
\text { Endoscopic retrograde cholangiopancreatography }=\text { ERCP. Intensive care Unit }=\text { ICU. } \text { Body mass index } \\
=\text { BMI. Acute kidney injury }=\text { AKI. Mild acute pancreatitis }=\text { MAP. Moderately severe acute pancreatitis = } \\
\text { MSAP. Severe acute pancreatitis = SAP. Odds ratio }=\text { OR. Endoscopic retrograde } \\
\text { cholangiopancreatography }=\text { ERCP. Pancreatic pseudocyst }=\text { PPC. Walled-off necrosis }=\text { WON. Acute } \\
\text { peripancreatic fluid collection = APFC. Acute necrosis collection = ANC. Percutaneous drainage = PCD. } \\
\text { Hypertriglyceridemia = HTG }\end{array}$} \\
\hline
\end{tabular}

Firstly, univariate analysis was performed to evaluate the risk factors of death. Age $\geq 80$ years old AP patients showed a higher death rate $(\mathrm{OR}, 1.058,95 \% \mathrm{Cl}, 1.019-1.098, \mathrm{P}=0.003), \mathrm{SAP}$, a more severe AP presentation(OR, $17.500,95 \% \mathrm{Cl}, 5.237-58.479, \mathrm{P}=0.001)$, the operative of $\mathrm{ERCP}(\mathrm{OR}, 0.217,95 \% \mathrm{Cl}$, $0.564-2.839, P=0.012)$, the presence of organ failure $(P=0.001)$ and local complication of ANC and ANC infection patients $(P=0.001)$ also showed a significant different clinical presentation. 
At the multivariate analysis, age over 80 years $(\mathrm{OR}, 3.299,95 \% \mathrm{Cl}, 1.316-8.269, \mathrm{P}=0.011)$, acute respiratory failure (OR, 4.266, 95\% Cl, 1.323-13.500, $\mathrm{P}=0.015)$, Shock (OR, 4.952, 95\% Cl, 1.575-15.569, $P=0.006)$, Multi-organ failure $(O R, 4.612,95 \% \mathrm{Cl}, 1.387-15.336, P=0.013)$ were all verified as independent risk factors for a higher mortality rate.

\section{ICU Admission (the Details Were Listed In Table 4)}


Table 4

Univariate and multivariate analysis of the risk factors on ICU admission in aging patients of acute pancreatitis

\begin{tabular}{|c|c|c|c|c|}
\hline \multirow[t]{2}{*}{ Variable } & \multicolumn{2}{|l|}{ Univariate analysis } & \multicolumn{2}{|l|}{ Multivariate analysis } \\
\hline & OR $(95 \% \mathrm{Cl})$ & P value & OR $(95 \% \mathrm{Cl})$ & $P$ value \\
\hline Age(years) & $1.228(0.745-2.203)$ & 0.402 & & \\
\hline Sex Male/ Female & $0.925(0.631-1.356)$ & 0.688 & & \\
\hline BMI & $1.128(1.055-1.206)$ & 0.001 & $0.989(0.901-1.086)$ & 0.812 \\
\hline \multicolumn{5}{|l|}{ Severity of AP } \\
\hline MAP & Reference & & Reference & \\
\hline MSAP & $1.825(0.965-3.453)$ & 0.064 & $1.231(0.571-2.654)$ & 0.596 \\
\hline SAP & $38.036(19.246-75.171)$ & 0.001 & $11.722(4.780-28.764)$ & 0.001 \\
\hline \multicolumn{5}{|l|}{ Etiology of AP } \\
\hline Biliary & Reference & & Reference & \\
\hline HTG & $0.553(0.071-4.283)$ & 0.026 & $2.111(0.517-8.624)$ & 0.298 \\
\hline Alcohol & $1.561(0.438-5.566)$ & 0.013 & $2.558(0.708-9.249)$ & 0.152 \\
\hline Others & $0.941(0.353-2.507)$ & 0.670 & $1.162(0.474-2.850)$ & 0.743 \\
\hline Hypertension & $1.641(1.107-2.431)$ & 0.014 & $1.445(0.825-2.531)$ & 0.198 \\
\hline Diabetes & $1.222(0.611-2.444)$ & 0.572 & & \\
\hline Chronic renal failure & $0.736(0.192-2.812)$ & 0.654 & & \\
\hline History of smoking & $0.895(0.559-1.433)$ & 0.643 & & \\
\hline History of drinking & $1.270(0.779-2.070)$ & 0.339 & & \\
\hline \multicolumn{5}{|l|}{ Symptoms } \\
\hline Abdominal pain & $2.218(1.475-3.336)$ & 0.001 & $1.906(1.052-3.453)$ & 0.033 \\
\hline Vomiting & $1.840(0.912-3.714)$ & 0.089 & & \\
\hline Diarrhea & $1.239(0.778-1.974)$ & 0.357 & & \\
\hline \multicolumn{5}{|c|}{$\begin{array}{l}\text { Abbreviation: Acute pancreatitis }=\text { AP. Acute respiratory failure }=\text { ARF. Length of stay }=\text { LOS. } \\
\text { Endoscopic retrograde cholangiopancreatography }=\text { ERCP. Intensive care Unit }=\text { ICU. Body mass index } \\
\text { = BMI. Acute kidney injury }=\text { AKI. Mild acute pancreatitis }=\text { MAP. Moderately severe acute pancreatitis }= \\
\text { MSAP. Severe acute pancreatitis = SAP. Odds ratio }=\text { OR. Endoscopic retrograde } \\
\text { cholangiopancreatography }=\text { ERCP. Pancreatic pseudocyst }=\text { PPC. Walled-off necrosis }=\text { WON. Acute } \\
\text { peripancreatic fluid collection = APFC. Acute necrosis collection = ANC. Percutaneous drainage = PCD. } \\
\text { Hypertriglyceridemia = HTG }\end{array}$} \\
\hline
\end{tabular}




\section{Variable}

Jaundice

Dyspnea

Operative treatment

\begin{tabular}{lllll} 
ERCP & $0.602(0.0 .368-0.985)$ & 0.043 & $1.335(0.690-2.585)$ & 0.391 \\
\hline PCD & $1.144(0.738-1.772)$ & 0.547 & & \\
Cholecystectomy & $0.514(0.168-1.576)$ & 0.245 & & \\
Necrosectomy & $2.386(1.159-4.915)$ & 0.018 & $0.840(0.247-2.861)$ & 0.780
\end{tabular}

Organ failure

Acute respiratory failure

18.413(10.906-31.087)

0.001

4.177(1.847-9.443)

0.001

Acute renal failure

14.655(6.962-30.849)

0.001

0.826(0.236-2.890)

0.765

Shock

3729(0.000-)

0.998

Multi-organ failure

26.928(9.439-76.823)

0.001

$5.103(1.270-20.503)$

0.022

Local complications

\begin{tabular}{lllll} 
ANC & $5.287(2.442-11.449)$ & 0.001 & $0.593(0.175-2.017)$ & 0.403 \\
\hline ANC infection & $2.615(1.640-4.170)$ & 0.001 & $3.097(0.680-14.105)$ & 0.144 \\
\hline APFC & $5.091(1.696-15.284)$ & 0.004 & $0.894(0.434-1.841)$ & 0.760 \\
\hline APFC infection & $22.909(7.631-68.778)$ & 0.001 & $3.146(0.730-13.544)$ & 0.124 \\
\hline WON & $3.818(0.750-19.441)$ & 0.107 & $1.096(0.087-13.810)$ & 0.944 \\
\hline WON infection & $1.273(0.130-12.475)$ & 0.836 & $1.805(0.158-20.634)$ & 0.635 \\
\hline PPC & $0.000(0.000)$ & 0.999 & $0.000(0.000-)$ & 0.999
\end{tabular}

Abbreviation: Acute pancreatitis $=$ AP. Acute respiratory failure $=$ ARF. Length of stay $=$ LOS.

Endoscopic retrograde cholangiopancreatography = ERCP. Intensive care Unit = ICU. Body mass index $=$ BMI. Acute kidney injury $=$ AKI. Mild acute pancreatitis $=$ MAP. Moderately severe acute pancreatitis $=$ MSAP. Severe acute pancreatitis $=$ SAP. Odds ratio $=$ OR. Endoscopic retrograde cholangiopancreatography $=$ ERCP. Pancreatic pseudocyst $=$ PPC. Walled-off necrosis $=$ WON. Acute peripancreatic fluid collection $=$ APFC. Acute necrosis collection $=$ ANC. Percutaneous drainage $=$ PCD. Hypertriglyceridemia $=$ HTG

$\mathrm{BMI}(\mathrm{OR}, 1.128,95 \% \mathrm{Cl}, 1.055-1.206, \mathrm{P}=0.001)$, SAP, a more severe AP presentation(OR, 38.036, 95\% Cl, $19.246-75.171, P=0.001)$, the etiology of hypertriglyceridemia(OR, $0.553,95 \% \mathrm{Cl}, 0.071-4.283, \mathrm{P}=$ 0.026), absence of a typical AP symptomatology of abdominal pain(OR, 2.218, 95\% Cl, 1.475-3.336, $\mathrm{P}=$ $0.001)$, Necrosectomy $(O R, 2.386,95 \% \mathrm{Cl}, 1.159-4.915, P=0.018)$, acute respiratory failure $(P=0.001)$ and local complication $(P=0.001)$ were significantly associated to a higher ICU admission rate at the 
univariate analysis. However, at the multivariate analysis, ICU admission was influenced by a more severe AP presentation(OR, $11.722,95 \% \mathrm{Cl}, 4.780-28.764, \mathrm{P}=0.001)$, absence of abdominal pain(OR, $1.906,95 \%$ $\mathrm{Cl}, 1.052-3.453, \mathrm{P}=0.033)$, acute respiratory failure $(\mathrm{OR}, 4.177,95 \% \mathrm{Cl}, 1.847-9.443, \mathrm{P}=0.001)$, Multiorgan failure(OR, $5.103,95 \% \mathrm{Cl}, 1.270-20.503, \mathrm{P}=0.022)$.

length of stay(LOS) (The details were listed in Table 5) 
Table 5

Univariate and multivariate analysis of the risk factors on length of stay in aging patients of acute pancreatitis

\begin{tabular}{|c|c|c|c|c|}
\hline \multirow[t]{2}{*}{ Variable } & \multicolumn{2}{|l|}{ Univariate analysis } & \multicolumn{2}{|l|}{ Multivariate analysis } \\
\hline & $\mathrm{B}(95 \% \mathrm{Cl})$ & $P$ value & $\mathrm{B}(95 \% \mathrm{Cl})$ & $P$ value \\
\hline Age(years) & $1.105(0.020-0.265)$ & 0.023 & $0.149(2.027-6.268)$ & 0.001 \\
\hline Sex Male/ Female & $0.092(0.032-3.802)$ & 0.046 & $0.093(0.336-3.542)$ & 0.018 \\
\hline BMI & $0.063(0.000-0.535)$ & 0.173 & & \\
\hline Severity of AP & $0.363(3.758-6.033)$ & 0.001 & $0.218(1.567-4.322)$ & 0.001 \\
\hline Etiology of AP & $0.009(-0.857-1.038)$ & 0.188 & & \\
\hline Hypertension & $0.095(0.112-4.031)$ & 0.038 & $0.060(-0.311-2.914)$ & 0.113 \\
\hline Diabetes & $0.026(-2.499-4.524)$ & 0.571 & & \\
\hline Chronic renal failure & $-0.009(-6.875-5.641)$ & 0.847 & & \\
\hline History of smoking & $-0.034(-3.168-1.432)$ & 0.459 & & \\
\hline History of drinking & $0.022(-1.880-3.055)$ & 0.640 & & \\
\hline \multicolumn{5}{|l|}{ Symptoms } \\
\hline Abdominal pain & $0.028(-1.323-2.508)$ & 0.544 & & \\
\hline Vomiting & $0.031(-2.384-4.917)$ & 0.496 & & \\
\hline Diarrhea & $0.150(1.560-6.198)$ & 0.001 & $0.073(-0.112-3.894)$ & 0.064 \\
\hline Jaundice & $0.137(1.618-7.750)$ & 0.003 & $0.080(0.146-5.324)$ & 0.038 \\
\hline Dyspnea & $1.367(2.160-7.533)$ & 0.001 & $0.071(-0.137-4.431)$ & 0.065 \\
\hline \multicolumn{5}{|l|}{ Operative treatment } \\
\hline ERCP & $0.001(-2.251-2.320)$ & 0.977 & & \\
\hline Percutaneous drainage & $0.215(3.063-7.332)$ & 0.001 & $0.205(5.336-11.676)$ & 0.001 \\
\hline Cholecystectomy & $-0.036(-6.710-2.892)$ & 0.435 & & \\
\hline Necrosectomy & $0.307(9.171-16.320)$ & 0.001 & $0.205(5.336-11.676)$ & 0.001 \\
\hline \multicolumn{5}{|c|}{$\begin{array}{l}\text { Abbreviation: Acute pancreatitis = AP. Acute respiratory failure = ARF. Length of stay = LOS. } \\
\text { Endoscopic retrograde cholangiopancreatography }=\text { ERCP. Intensive care Unit = ICU. Body mass index } \\
\text { = BMI. Acute kidney injury = AKI. Mild acute pancreatitis = MAP. Moderately severe acute pancreatitis = } \\
\text { MSAP. Severe acute pancreatitis = SAP. Odds ratio = OR. Endoscopic retrograde } \\
\text { cholangiopancreatography = ERCP. Pancreatic pseudocyst }=\text { PPC. Walled-off necrosis }=\text { WON. Acute } \\
\text { peripancreatic fluid collection = APFC. Acute necrosis collection = ANC. Percutaneous drainage = PCD. } \\
\text { Hypertriglyceridemia = HTG }\end{array}$} \\
\hline
\end{tabular}




\begin{tabular}{|c|c|c|c|c|}
\hline Variable & \multicolumn{2}{|l|}{ Univariate analysis } & \multicolumn{2}{|l|}{ Multivariate analysis } \\
\hline \multicolumn{5}{|l|}{ Organ failure } \\
\hline Acute respiratory failure & $0.347(6.277-10.343)$ & 0.001 & $0.143(0.810-6.025)$ & 0.010 \\
\hline Acute renal failure & $0.224(4.328-9.977)$ & 0.001 & $0.021(-3.137-4.455)$ & 0.733 \\
\hline Shock & $0.301(10.377-18.743)$ & 0.001 & $0.229(6.930-15.216)$ & 0.001 \\
\hline Multi-organ failure & $0.136(1.662-8.031)$ & 0.003 & $-0.181(-10.425-2.450)$ & 0.200 \\
\hline Local complication & $0.282(1.427-2.696)$ & 0.001 & $0.073(-0.090-1.160)$ & 0.093 \\
\hline \multicolumn{5}{|c|}{$\begin{array}{l}\text { Abbreviation: Acute pancreatitis }=\text { AP. Acute respiratory failure }=\text { ARF. Length of stay }=\text { LOS. } \\
\text { Endoscopic retrograde cholangiopancreatography }=\text { ERCP. Intensive care Unit }=\text { ICU. } \text { Body mass index } \\
=\text { BMI. Acute kidney injury }=\text { AKI. Mild acute pancreatitis }=\text { MAP. Moderately severe acute pancreatitis }= \\
\text { MSAP. Severe acute pancreatitis = SAP. Odds ratio }=\text { OR. Endoscopic retrograde } \\
\text { cholangiopancreatography }=\text { ERCP. Pancreatic pseudocyst }=\text { PPC. Walled-off necrosis }=\text { WON. Acute } \\
\text { peripancreatic fluid collection = APFC. Acute necrosis collection }=\text { ANC. Percutaneous drainage = PCD. } \\
\text { Hypertriglyceridemia }=\text { HTG }\end{array}$} \\
\hline
\end{tabular}

With regards to LOS, There were many rick factors related to LOS by univariate analysis like age(OR, $1.105,95 \% \mathrm{Cl}, 0.020-0.265, \mathrm{P}=0.023)$, Sex (OR, 0.092, 95\% Cl, 0.032-3.802, $\mathrm{P}=0.046)$, Severity of AP (OR, $0.363,95 \% \mathrm{Cl}, 3.758-6.033, \mathrm{P}=0.001$ ), hypertension (OR, 0.095, 95\% Cl, 0.112-4.031, $\mathrm{P}=0.038$ ), diarrhea (OR, 0.150, 95\% Cl, 1.560-6.198, P = 0.001), Jaundice(OR, 0.137, 95\% Cl, 1.618-7.750, $\mathrm{P}=$ 0.003), dyspnea (OR, 1.367, 95\% Cl, 2.160-7.533, $\mathrm{P}=0.001)$, $\mathrm{PCD}(\mathrm{OR}, 0.215,95 \% \mathrm{Cl}, 3.063-7.332, \mathrm{P}=$ 0.001), Necrosectomy $(\mathrm{OR}, 0.307,95 \% \mathrm{Cl}, 9.171-16.320, \mathrm{P}=0.001)$, organ failure $(\mathrm{P}=0.001)$ and local complication $(\mathrm{OR}, 0.282,95 \% \mathrm{Cl}, 1.427-2.696, \mathrm{P}=0.001)$. At the multivariate analysis, age $(\mathrm{OR}, 0.149$, $95 \% \mathrm{Cl}, 2.027-6.268, \mathrm{P}=0.001)$, female $(\mathrm{OR}, 0.093,95 \% \mathrm{Cl}, 0.336-3.542, \mathrm{P}=0.018)$, Severity of AP (OR, 0. $0.218,95 \% \mathrm{Cl}, 1.567-4.322, \mathrm{P}=0.001)$, diarrhea (OR, $0.073,95 \% \mathrm{Cl},-0.112-3.894, \mathrm{P}=0.064)$, jaundice(OR, $0.080,95 \% \mathrm{Cl}, 0.146-5.324, \mathrm{P}=0.038), \mathrm{PCD}(\mathrm{OR}, 0.205,95 \% \mathrm{Cl}, 5.336-11.676, \mathrm{P}=0.001)$, necrosectomy $(\mathrm{OR}, 0.205,95 \% \mathrm{Cl}, 5.336-11.676, \mathrm{P}=0.001)$, acute respiratory failure $(\mathrm{OR}, 0.143,95 \% \mathrm{Cl}$, $0.810-6.025, \mathrm{P}=0.010)$, shock $(\mathrm{OR}, 0.229,95 \% \mathrm{Cl}, 6.930-15.216, \mathrm{P}=0.001)$ were the independent risk factors for longer length of stay.

\section{Discussion}

With an great increase of aging population all over the world and the prolongation of life span, a great number of age-associated acute and chronic diseases has been occurred. AP was one of the acute diseases and the incidence rate increases proportionally with the increasing age[25]. Data from an emergency departments(ED) presented that aging patients aged over 65 years old diagnosed with AP reached one-third of all patients[8]. Another register study reported that a six-fold increase in the incidence rate from AP patients aged $0-49$ years to patients over 80 years during a decade[26]. 
Despite we have paid attention to the importance of clinical outcomes of the frailer portion of population of AP, but it is only a small part of researched with limited data and disputable results are present in those articles. A study from European published that the morbidity rate of AP aging patients aged $\geq 65$ years was increased and especially among the aging patients $\geq 80$ years[4]. However, Kim JE.el proposed that the clinical courses of AP between older and younger patients have not evidenced any significant difference[15]. The difference between those research probably due to the age bracket set by the different authors. Actually, based on those study, they mostly make a conclusion that aging patients generally developed to a more severe AP and age may regarded as a risk factor for the clinical outcomes. With the purpose of giving a more contribution to this still controversial issue, we compared the clinical outcomes of the aging AP patients divided into older group (60-79 years old) and oldest group ( $\geq 80$ years old) who admitted to our pancreas centre in the first affiliated hospital of Nanchang university of China. Given the increase of life expectancy of aging patients in China, the cut-off chosen of age in our study will represent a large proportion of the aging patients in the near future.

In our retrospective study, we aimed at analyzing the age-associated clinical outcomes and assessed the risk factors of aging patients in the disease. Interestingly, our study figured out aging patients aged over 80 years old significantly influenced the mortality rate and length of hospitalization, while the ICU admission rate was similar to the older group.

With the clinical presentation of aging AP patients, more severe manifestations was found in the oldest group as compared to the older group in our study. The phenomenon has also been evidenced by previous research that increasing age was closely related to a more severe clinical course of AP[15-18, 27]. The underlying mechanism of the above condition is still debate. A view proposed in a literature that the increasing age was associated to the loss of protective proteins of AP which led an adverse clinical course in oldest patients of the disease[28]. Pre-existing comorbidities such as diabetes, chronic renal disease would influence the physiological functions of aging AP patients which causing a more severe inflammatory response reflected as primary clinical manifestation of the disease[13].

According to clinical experiences, the clinical assessment of aging patients is usually more harder than younger patients[29]. and it is verified in our research that the absence of typical symptoms in our aging patients was $28 \%$ in all enrolled patients. It reminds us that increased awareness in aging AP patients should be given, especially for the more severe AP patients. In our study, we found that abnormal symptomatology was show in the aging AP patients. At this regard, a less frequent abdominal pain was evidenced in the oldest group rather than the older group which is in line with previous studies who report a decline of abdominal pain in aging patients over 80 years old. It may linked to a greater capacity to endure the pain and less feedbacks. In our study, we found that the abdominal pain was significant related to the ICU admission rate in aging patients for the first time. While the clinical symptom was not associated to the death rate which was not in line with previous study presented that the death rate is closely related to the abdominal pain[13]. We speculated that the phenomenon evidenced in our research was attributed to the fully treatment experience for more severe aging AP patients in our hospital, which is one of the largest pancreas centre in China, leading a higher ICU admission rate and lower death rate. 
Besides, an interesting phenomenon of the symptoms was evidenced in our study that dyspnea, jaundice and diarrhea presented more frequent in oldest patients as compared to older group $(P<0.05)$. The relation between AP and dyspnea symptoms in aging patients has been already reported[22]. While the other two symptoms of jaundice and diarrhea was not in line with previous research which reported that those symptoms was similar in older and oldest group. We suspected that age itself was the explanation regard to diversity of symptoms in aging people of AP. In addition, our study also surprised founds that the of symptom of jaundice is related to LOS which have not reported by other studies. The conditions reminder us that we should pay more attention to the symptoms of diarrhea, jaundice, dyspnea in the absence of abdominal pain of AP in aging patients in the future which bring lower death rate, ICU admission rate and shorter LOS.

A research have reported that lower $\mathrm{BMI}$ ranged 18.5 to 19.9 had a higher risk of death rate relative to those population with a BMI over 21.5[30]. Another study finds that BMI less than 20.0 is associated with an increased risk of death, whereas a $\mathrm{BMI} \geq 23.0$ is not associated with higher death rate[31]. In our study, lower BMI $(21.07 \pm 3.18)$ was evidenced in oldest as group compared to older group $(22.36 \pm 2.89)$ and there were significant difference in the two group. Although the BMI is not the independent risk factor of death rate in our study, the BMI related age is associated to dying. One potential explanation for this may relate to energy and nutritional reserves. Malnutrition is verified a predictor of death and thin people with less energy and nutritional are less resistant to infection[32]. Hence, aging patients should take appropriate nutrition to increase their BMI to resist the disease.

Over expression of interleukin (IL)-6, IL-8 and tumor necrosis factor (TNF)-a was verified after AP onset as an already known proinflammatory status. The proinflammatory facilitates systemic symptoms, especially respiratory and renal injury as first manifestation of AP. In particular, IL-6, TNF-a and the alveolar macrophages were accumulated after the neutrophils activated in the lung, then develop a kind of second wave of inflammation causing the onset of respiratory symptoms as first expression[33]. According to the conditions, it is necessary to provide intensive care, guarantee an enough monitoring of cardiopulmonary and a more suitable electrolytes and fluid replacement for the aging patients after AP onset[8]. With this regard, we found that a higher rate of ICU admission in the oldest group compared to older group and age $\geq 80$ years was an independent risk factors for ICU admission rate.

In regard to the etiology of AP, the most common cause of AP was biliary in aging patients which was much higher percentage in younger AP patients. But the proportion of biliary pancreatitis in the etiology of AP was no significant difference in older group and oldest group. The result was in line with previous researches that the higher incidence rate of gallstones-inducing AP was related to the increasing age[5]. In addition, we found that alcohol-induced AP and HTG-induced AP were less frequently encountered in aging patients. Previous study have been reported that alcoholic AP occurred more often in younger subjects[5]. While HTG, a common etiology of AP in China which accounts for $40 \%$ of all AP patients, have been decline to a small proportion of the etiology of AP. We speculated that the phenomenon of more frequent gallstone-induced AP in aging patients was associated to a more frequent disease of gallstones, with an much more thickening diameter of the common bile duct, which make aging patients 
more liable to the onset of biliary-induced AP[34, 35]. Although a literature stated a more severe clinical course among the disease of biliary-induced AP[36], we did not discover the relationship between gallstones-induced pancreatitis and mortality, ICU admission, or LOS in the multivariate logistic analysis in our aging AP patients. It is in line with the hypothesis which has been confirmed that the worst clinical outcomes in the aging AP patients would be mainly explained by other risk factors rather than etiology[37].

Regarding to the operative treatment, ERCP was the most common operation performed in the aging patients in our study. A similar rate of operative treatment of ERCP has been verified between the two old group. The phenomenon is caused by the proportion of the etiology of biliary. Besides, an interesting result was showed in our study that 19 patients aged 60-79 years old performed cholecystectomy but no patients aged $\geq 80$ years old underwent the operation. Previous study declared that the operation of cholecystectomy performed in hospitalization vastly reduce the risk of recurrence of AP which may related to a higher rate of infectious complications[13]. Another research reported that higher complication rate in aging patients aged $\geq 80$ years old after cholecystectomy with complicated biliary disease compared to those with an uncomplicated gallstones disease[38]. What is more, a view was proposed by some authors that cholecystectomy in AP patients $\geq 80$ years old increase the death rate eventually[39]. The discrepancy of the treatment of cholecystectomy in aging patients reflects the trend of avoiding operative treatment who was defined as not suitable to the operation[16]. Actually, It was conformed to Chinese conditions that aging patients often refuse surgical procedures which can alleviate the disease in hospital. They afraid the poor prognosis, high infection and mortality. Finally, in our analysis, all of operative treatment were not influence the mortality rate and ICU admission rate. While PCD and necrosectomy of the operative treatment was evidenced the independent risk factors of LOS in aging patients aged $\geq 80$ years old.

The relationship between increasing age and death rate of AP was an important finding in our study. An obvious higher rate of death was certificated in oldest group as compared to the older group. What is more, we also found age $\geq 80$ years old is an independent risk factor of death. The other risk factor was organ failure includes acute respiratory failure, shock and multi-organ failure. It is in line with previous opinion that increasing age is closely related with a higher mortality rate of AP[17-20,34,35, 40]. We predicted the higher death rate of aging AP patients in oldest group was mainly attributed to the remarkable fragility of aging patients and a more severe clinical course of AP because the aging patients in the two group presented the similar rate of complications and operative treatment. Interestingly, our research reported that the death rate was $10.1 \%$ in all aging patients which is less than other studies. While previous study found that the death rate reached up to $20 \%$ in older patients aged over 55 years old $[34,41]$. Another literature proposed the clinical course of AP patients aged $\geq 80$ years was evidenced the death rate up to $25 \%[16,41]$. In addition, a lower death rate $(7.1 \%)$ was evidenced in recent study in older patients over 65 years old[13]. The discrepancy of death rate in our research and others was associated to the early diagnosis and the different proportion of severity of the disease. 


\section{Study Limitations}

Although the largest number of aging AP patients aged $\geq 80$ years old in our hospital of china was enrolled in our study which focuses on the AP clinical course and we represented the common management of those patients, some limitations should be listed. Firstly, it is a retrospectively study contains potential biases in study design. Secondly, it is not a multicentre study in different tertiary hospitals which presented the clinical traits in all aging AP patients in China.

\section{Conclusion}

Biliary pancreatitis is the main etiology of AP in aging patients especially aged $\geq 80$ years old. There is a closely relationship between increasing age and death rate and LOS. In addition, more attention should be given to the clinical symptoms in this frailer portion of the population. Based on our finding of this study, we proposed that a more comprehensive and goal-directed attendant diagnostic procedures should be done to early detect the disease and improve the outcomes in aging patients.

\section{Abbreviations}

Acute pancreatitis=AP. White blood cell=WBC. Lactic dehydrogenase=LDH. Alanine Aminotransferase $=$ ALT. Aspartate transaminase $=$ AST. Total bilirubin $=$ TBIL. . Direct bilirubin $=$ DBIL. $C-$ reactive protein $=\mathrm{CRP}$. Urea nitrogen $=\mathrm{BUN}$. Glucose $=\mathrm{GLU}$. Total cholesterol=TC. Triglyceride $=\mathrm{TG}$. Albumin=ALB. Amylase=AMY. Creatinine $=\mathrm{Cr}$. Acute Physiology and Chronic Health Evaluation II=APACHEII. Bedside index for severity in acute pancreatitis=BISAP. Hypertriglyceridemia=HTG. Acute respiratory failure $=A R F$. Length of stay $=$ LOS. Endoscopic retrograde cholangiopancreatography $=E R C P$. Intensive care Unit=ICU. Body mass index =BMI. Acute kidney injury=AKI. Mild acute pancreatitis=MAP. Moderately severe acute pancreatitis=MSAP. Severe acute pancreatitis $=S A P$. Odds ratio $=O R$. Endoscopic retrograde cholangiopancreatography=ERCP. Pancreatic pseudocyst=PPC. Walled-off necrosis=WON. Acute peripancreatic fluid collection=APFC. Acute necrosis collection=ANC. Percutaneous drainage=PCD. Hypertriglyceridemia $=H T G$. Odds ratio=OR. Computed tomographic $=$ CT.

\section{Declarations}

Acknowledgments $\square$ We thank Dr. Fengwen Xie for helpful suggestions in data collection.

Authors' contributions: YuBingjun conceived the study; Fengwen Xie, Nonghua Lu participated in the study design; YuBingjun collected the data; YuBingjun, Wenhua He, Cong He and Nianshuang Li performed the statistical analyses; YuBingjun drafted the manuscript; Nonghua Lu edited and checked the manuscript. All of the authors have read and approved the final manuscript.

Funding $\square$ The study design and data collection were funded by the national natural science foundation of China: (No :81660114 and 81860122). The Key Research and Development Program from the Science and Technology Department of Jiangxi Province (No. 20192BBG70037). 
Availability of data and materials: The data enrolled and analyzed in our study are not publicly available due to appropriate protection of patient personal information but are available from the corresponding author on reasonable request.

Ethics approval and consent to participate: The study was approved by the ethics committee of the First Affiliated Hospital of Nanchang University (database approval number: 2011001) prior to data collection. All procedures followed were in accordance with the ethical standards of the responsible committee on human experimentation (institutional and national) and with the Helsinki Declaration of 1975, as revised in 2008. This study is a retrospective analysis, it did not include any human trial, and as such no informed consent from patients was needed.

Consent for publication: Not applicable.

Competing interests: The authors declare no potential conflicts of interest. No writing assistance was provided in the production of this manuscript.

Patient and public involvementaPatients were not involved in the design of this research study.

Author details: ${ }^{1}$ Department of Gastroenterology, The First Affiliated Hospital of Nanchang University, Nanchang 330006, Jiangxi Province, China. ${ }^{2}$ Department of cardiothoracic surgery, The First Affiliated Hospital of Nanchang University, Nanchang 330006, Jiangxi Province, China

\section{References}

1 https://ec.europa.eu/eurostat/statistics-explained/index.php? title=Population_structure_and_ageing. Accessed April 1, 2019. .

2 Gluszek S and Koziel D. Prevalence and progression of acute pancreatitis in the Swietokrzyskie Voivodeship population. Pol Przegl Chir. 2012; 84(12):618-625.

3 Yadav D and Lowenfels AB. Trends in the epidemiology of the first attack of acute pancreatitis: a systematic review. PANCREAS. 2006; 33(4):323-330.

4 Roberts SE, Morrison-Rees S, John A, Williams JG, Brown TH and Samuel DG. The incidence and aetiology of acute pancreatitis across Europe. PANCREATOLOGY. 2017; 17(2):155-165.

$5 \quad$ Yadav D and Lowenfels AB. The epidemiology of pancreatitis and pancreatic cancer. GASTROENTEROLOGY. 2013; 144(6):1252-1261.

6 Carr RA, Rejowski BJ, Cote GA, Pitt HA and Zyromski NJ. Systematic review of hypertriglyceridemia-induced acute pancreatitis: A more virulent etiology? PANCREATOLOGY. 2016; 16(4):469-476. 
7 Scherer J, Singh VP, Pitchumoni CS and Yadav D. Issues in hypertriglyceridemic pancreatitis: an update. J CLIN GASTROENTEROL. 2014; 48(3):195-203.

8 Gloor B, Ahmed Z, Uhl W and Buchler MW. Pancreatic disease in the elderly. Best Pract Res Clin Gastroenterol. 2002; 16(1):159-170.

9 Neoptolemos JP, Raraty M, Finch M and Sutton R. Acute pancreatitis: the substantial human and financial costs. GUT. 1998; 42(6):886-891.

10 Banks PA and Freeman ML. Practice guidelines in acute pancreatitis. AM J GASTROENTEROL. 2006; 101(10):2379-2400.

11 Swaroop VS, Chari ST and Clain JE. Severe acute pancreatitis. JAMA. 2004; 291(23):2865-2868.

12 Whitcomb DC. Clinical practice. Acute pancreatitis. N Engl J Med. 2006; 354(20):2142-2150.

13 Quero G, Covino M, Ojetti V, Fiorillo C, Rosa F, Menghi R, Laterza V, Candelli M, Franceschi F and Alfieri S. Acute pancreatitis in oldest old: a 10-year retrospective analysis of patients referred to the emergency department of a large tertiary hospital. Eur J Gastroenterol Hepatol. 2020; 32(2):159-165.

14 Gullo L, Migliori M, Olah A, Farkas G, Levy P, Arvanitakis C, Lankisch P and Beger H. Acute pancreatitis in five European countries: etiology and mortality. PANCREAS. 2002; 24(3):223-227.

15 Kim JE, Hwang JH, Lee SH, Cha BH, Park YS, Kim JW, Jeong SH, Kim N and Lee DH. The clinical outcome of elderly patients with acute pancreatitis is not different in spite of the different etiologies and severity. Arch Gerontol Geriatr. 2012; 54(1):256-260.

16 Somasekar K, Foulkes R, Morris-Stiff G and Hassn A. Acute pancreatitis in the elderly - Can we perform better? Surgeon. 2011; 9(6):305-308.

17 Xin MJ, Chen H, Luo B and Sun JB. Severe acute pancreatitis in the elderly: etiology and clinical characteristics. World J Gastroenterol. 2008; 14(16):2517-2521.

18 de Beaux AC, Palmer KR and Carter DC. Factors influencing morbidity and mortality in acute pancreatitis; an analysis of 279 cases. GUT. 1995; 37(1):121-126.

19 Gardner TB, Vege SS, Chari ST, Pearson RK, Clain JE, Topazian MD, Levy MJ and Petersen BT. The effect of age on hospital outcomes in severe acute pancreatitis. PANCREATOLOGY. 2008; 8(3):265-270.

20 Miki C, Kusunoki M, Inoue Y, Uchida K, Mohri Y, Buckels JA and McMaster P. Remodeling of the immunoinflammatory network system in elderly cancer patients: implications of inflamm-aging and tumor-specific hyperinflammation. SURG TODAY. 2008; 38(10):873-878.

21 Xin MJ, Chen H, Luo B and Sun JB. Severe acute pancreatitis in the elderly: etiology and clinical characteristics. World J Gastroenterol. 2008; 14(16):2517-2521. 
22 Quero G, Covino M, Fiorillo C, Rosa F, Menghi R, Simeoni B, Potenza A, Ojetti V, Alfieri S and Franceschi F. Acute pancreatitis in elderly patients: a single-center retrospective evaluation of clinical outcomes. Scand J Gastroenterol. 2019; 54(4):492-498.

23 Banks PA, Bollen TL, Dervenis C, Gooszen HG, Johnson CD, Sarr MG, Tsiotos GG and Vege SS. Classification of acute pancreatitis-2012: revision of the Atlanta classification and definitions by international consensus. GUT. 2013; 62(1):102-111.

24 Li W, Zhang L, Li J, Dellinger EP, Forsmark CE, Layer P, L'Evy P, Poma EM, Petrov MS, Shimosegawa T, Siriwardena AK, Uomo G, Whitcomb DC and Windsor JA. [Determinant-based classification of acute pancreatitis severity: an international multidisciplinary consultation: the 2013 Chinese edition]. Zhonghua Wai Ke Za Zhi. 2014; 52(5):321-324.

25 Schepers NJ, Bakker OJ, Besselink MG, Bollen TL, Dijkgraaf MG, van Eijck CH, Fockens P, van Geenen EJ, van Grinsven J, Hallensleben ND, Hansen BE, van Santvoort HC, Timmer R, Anten MP, Bolwerk $\mathrm{CJ}$ and van Delft $\mathrm{F}$, et al. Early biliary decompression versus conservative treatment in acute biliary pancreatitis (APEC trial): study protocol for a randomized controlled trial. TRIALS. 2016; 17:5.

26 Sandzen B, Rosenmuller M, Haapamaki MM, Nilsson E, Stenlund HC and Oman M. First attack of acute pancreatitis in Sweden 1988 - 2003: incidence, aetiological classification, procedures and mortality - a register study. BMC GASTROENTEROL. 2009; 9:18.

27 Fu S, Stanek A, Mueller CM, Brown NA, Huan C, Bluth MH and Zenilman ME. Acute pancreatitis in aging animals: loss of pancreatitis-associated protein protection? World J Gastroenterol. 2012; 18(26):3379-3388.

28 Coelho A, Machado M, Sampietre SN, Da SF, Cunha J and D'Albuquerque L. Local and systemic effects of aging on acute pancreatitis. PANCREATOLOGY. 2019; 19(5):638-645.

29 Covino M, Petruzziello C, Onder G, Migneco A, Simeoni B, Franceschi F and Ojetti V. A 12-year retrospective analysis of differences between elderly and oldest old patients referred to the emergency department of a large tertiary hospital. MATURITAS. 2019; 120:7-11.

30 Murayama H, Liang J, Shaw BA, Botoseneanu A, Kobayashi E, Fukaya T and Shinkai S. Age and gender differences in the association between body mass index and all-cause mortality among older Japanese. Ethn Health. 2018:1-14.

31 Tamakoshi A, Yatsuya H, Lin Y, Tamakoshi K, Kondo T, Suzuki S, Yagyu K and Kikuchi S. BMI and all-cause mortality among Japanese older adults: findings from the Japan collaborative cohort study. Obesity (Silver Spring). 2010; 18(2):362-369.

32 Sahyoun NR, Jacques PF, Dallal G and Russell RM. Use of albumin as a predictor of mortality in community dwelling and institutionalized elderly populations. J CLIN EPIDEMIOL. 1996; 49(9):981-988. 
33 Closa D, Sabater L, Fernandez-Cruz L, Prats N, Gelpi E and Rosello-Catafau J. Activation of alveolar macrophages in lung injury associated with experimental acute pancreatitis is mediated by the liver. ANN SURG. 1999; 229(2):230-236.

34 Malik AM. Biliary pancreatitis. Deadly threat to the elderly. Is it a real threat? Int J Health Sci (Qassim). 2015; 9(1):35-39.

35 Turnbull IR, Clark AT, Stromberg PE, Dixon DJ, Woolsey CA, Davis CG, Hotchkiss RS, Buchman TG and Coopersmith CM. Effects of aging on the immunopathologic response to sepsis. CRIT CARE MED. 2009; 37(3):1018-1023.

36 Zheng Y, Zhou Z, Li H, Li J, Li A, Ma B, Zhang T, Liao Q, Ye Y, Zhang Z, Yang Y, Wang Z, Zhang Z, Yang $\mathrm{J}$ and Li F. A multicenter study on etiology of acute pancreatitis in Beijing during 5 years. PANCREAS. 2015; 44(3):409-414.

37 Losurdo G, lannone A, Principi M, Barone M, Ranaldo N, lerardi E and Di Leo A. Acute pancreatitis in elderly patients: A retrospective evaluation at hospital admission. EUR J INTERN MED. 2016; 30:88-93.

38 Yu W, Hu S, Qi Y and Li B. The correlation between sonographic diagnosis and laparoscopic observations on fatty liver. J Laparoendosc Adv Surg Tech A. 2009; 19(2):163-169.

39 Tambyraja AL, Kumar S and Nixon SJ. Outcome of laparoscopic cholecystectomy in patients 80 years and older. WORLD J SURG. 2004; 28(8):745-748.

40 Starr ME, Ueda J, Yamamoto S, Evers BM and Saito H. The effects of aging on pulmonary oxidative damage, protein nitration, and extracellular superoxide dismutase down-regulation during systemic inflammation. Free Radic Biol Med. 2011; 50(2):371-380.

41 Koziel D, Gluszek-Osuch M, Suliga E, Zak M and Gluszek S. Elderly persons with acute pancreatitis - specifics of the clinical course of the disease. CLIN INTERV AGING. 2019; 14:33-41.

\section{Figures}




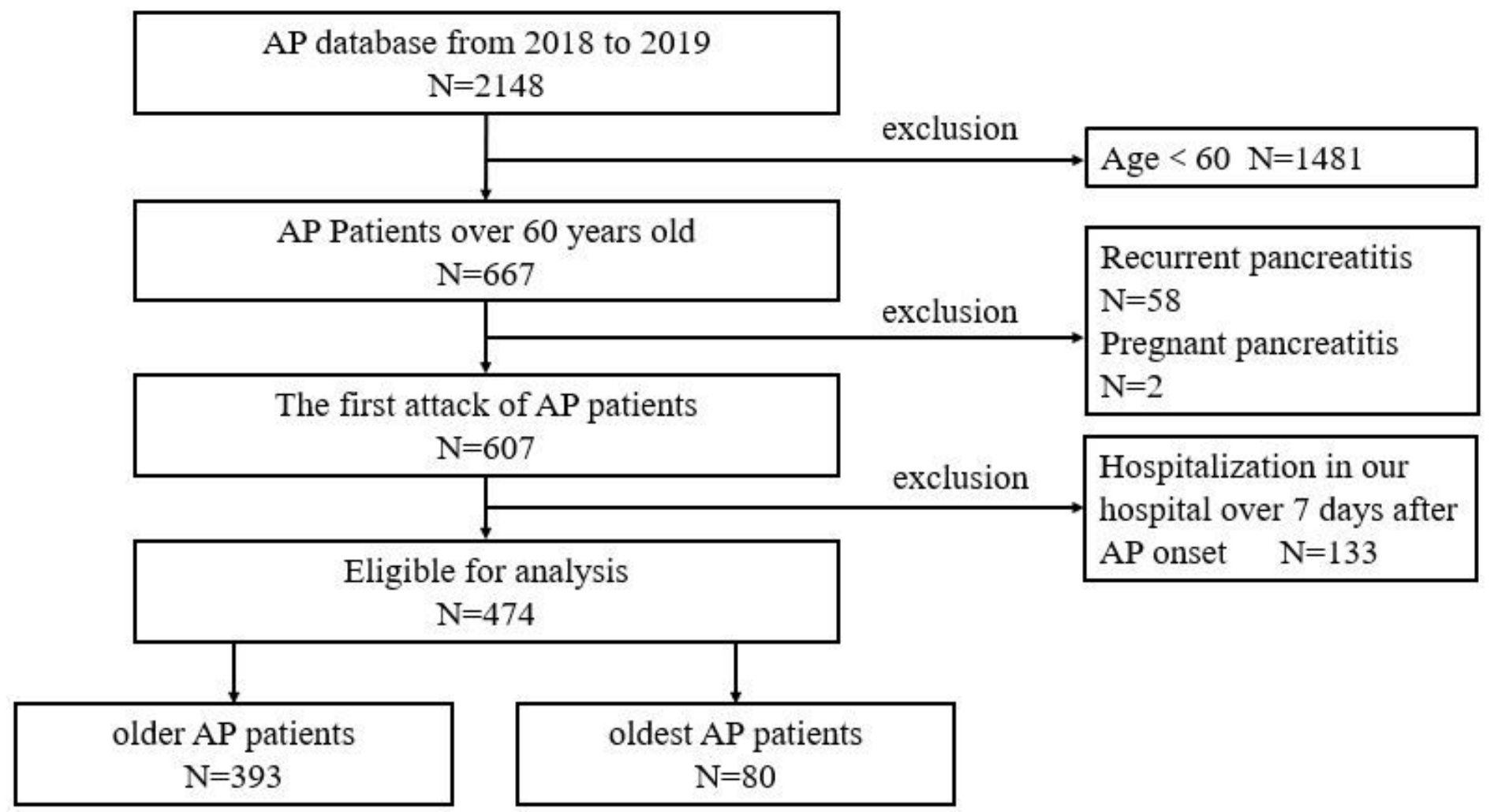

Figure 1

Flow chart of study participants

\section{Supplementary Files}

This is a list of supplementary files associated with this preprint. Click to download.

- trialsSTROBEchecklistcohort.docx

- SupplementaryTable1.docx 